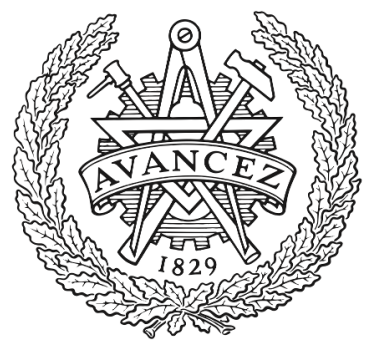

CHALMERS

UNIVERSITY OF TECHNOLOGY

\title{
Phase Distribution Optimization for 1-Bit Transmitarrays with Near-Field Coupling Feeding Technique
}

Downloaded from: https://research.chalmers.se, 2023-04-26 08:52 UTC

Citation for the original published paper (version of record):

Vilenskiy, A., Makurin, M., Lee, C. (2020). Phase Distribution Optimization for 1-Bit Transmitarrays with Near-Field Coupling Feeding

Technique. 14th European Conference on Antennas and Propagation, EuCAP 2020.

http://dx.doi.org/10.23919/EuCAP48036.2020.9135564

N.B. When citing this work, cite the original published paper. 


\title{
Phase Distribution Optimization for 1-Bit Transmitarrays with Near-Field Coupling Feeding Technique
}

\author{
Artem R. Vilenskiy ${ }^{1}$, Mikhail N. Makurin ${ }^{1}$, Chongmin Lee ${ }^{2}$ \\ ${ }^{1}$ RF Sensor Lab, Samsung Research Institute Russia, Moscow, Russia, temaforyou@yandex.ru \\ ${ }^{2}$ Smart Device Team, Samsung Research, Seoul, South Korea
}

\begin{abstract}
The paper presents a consideration of the optimum initial phase distribution for 1-bit transmitarrays with near-field coupling feeding technique. The study is based on the array factor decomposition into a series of continuous aperture distributions, which naturally includes the phase quantization errors. The previously proposed virtual focus approach is compared with the optimum quadratic initial phase distribution. Both methods are found to be very similar for specific values of distribution parameters in terms of farfield performance. Some further sidelobes level improvement is proposed.
\end{abstract}

Index Terms - transmitarray, phase quantization, sidelobes.

\section{INTRODUCTION}

Transmitarray (TA) technology has been actively employed during the last decade for the development of high-efficient beam-steering antenna systems of $\mathrm{cm}$ - and mm-wave frequencies [1] - [5]. Specific attention is being paid to the reconfigurable TAs with the 1-bit phase quantization [2], [4], [5]. Such structures are capable to provide beam steering with minimum insertion loss and design complexity, which is vital for many high-frequency systems. The main drawback of the conventional TAs with a focal source is a substantial longitudinal size [1]. To resolve this problem, recently we proposed a new architecture - the TA with near-field coupling to the fixed-beam antenna array. The block diagram of this architecture is presented in Fig. 1. In this case, the TA is excited by the radiative near field (plane wave) of the fixed-beam array. This excitation of the conventional 1-bit arrays leads to the generation of the parasitic mirror lobes - the particular case of the phase quantization sidelobes [6]. Numerous techniques have been previously proposed to reduce the negative phase quantization effects. In [6], it was mentioned that adding the

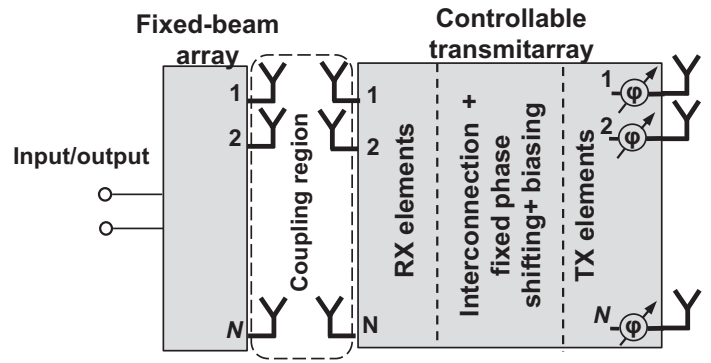

Fig. 1. The block diagram of the TA architecture with near-field coupling. initial phase distribution similar to the focal source excitation can reduce the sidelobes level (SLL). Below, this method will be called the "virtual focus approach". Different phase randomization methods were considered in [7], including the phase-added method. A useful approach for radar systems was presented in [8]. Despite the demonstrated SLL improvement, little or no papers exist that could show and verify the optimum initial phase distribution. The present study aims to contribute to the consideration of this problem by applying a useful array factor (AF) representation [9], which is given in Section II. With this approach, in Section III, we will try to formulate and verify an optimum initial phase synthesis procedure.

\section{TRANSMITARRAY RADIATION MODEL}

In this section, we will develop a radiation model of the TA using the aperture diffraction theory with some useful representation of AF. The object of the subsequent analysis is a TX part of the planar TA (Fig. 1) comprising a rectangular periodic array of $N_{x} \times N_{y}$ radiating elements with inter-element spacings $d_{x}$ and $d_{y}$.

\section{A. Representation of the Far Field}

In the far-field region, a TA of substantial electrical sizes can be conveniently and precisely approximated by a planar radiating aperture [10]. In this case, we can represent an aperture field distribution $\left(\mathbf{E}_{a}, \mathbf{H}_{a}\right)$ with the 1-bit phase quantization by a piecewise constant function:

$$
\begin{aligned}
& \mathbf{E}_{a}(x, y)=\mathbf{x}^{0} E_{0} \exp \left(j \varphi_{i j}^{\Sigma}\right), \quad x \in\left[x_{i}-\frac{d_{x}}{2} ; x_{i}+\frac{d_{x}}{2}\right), \\
& \mathbf{H}_{a}(x, y)=\mathbf{y}^{0} \frac{E_{0}}{\eta_{0}} \exp \left(j \varphi_{i j}^{\Sigma}\right), y \in\left[y_{j}-\frac{d_{y}}{2} ; y_{j}+\frac{d_{y}}{2}\right),
\end{aligned}
$$

where $E_{0}$ is a constant electric field magnitude; $\eta_{0}=120 \pi \Omega$; $\varphi^{\Sigma_{i j}}$ is $(i, j)$ element's excitation phase; $x_{i}, y_{j}$ are center coordinates of $(i, j)$ element; $i=1 \ldots N_{x}, j=1 \ldots N_{y}$.

Substituting (1) into the representation of the aperture far field $(\mathbf{E})[10]$, we can derive the following equation: 


$$
\begin{gathered}
\mathbf{E}(R, \theta, \varphi)=\frac{j k \exp (-j k R)}{2 \pi R} \frac{1+\cos (\theta)}{2} F_{s f}(\theta, \varphi) . \\
\cdot\left[\cos (\varphi) \theta^{0}-\sin (\varphi) \varphi^{0}\right] \\
F_{s f}(\theta, \varphi)=d_{x} d_{y} \operatorname{sinc}\left(k_{x} d_{x} / 2\right) \operatorname{sinc}\left(k_{y} d_{y} / 2\right) \mathrm{AF}, \\
\mathrm{AF}=\sum_{i=1}^{N_{x}} \sum_{j=1}^{N_{y}} \exp \left(j\left[\Phi_{i j}+\varphi_{i j}^{\Sigma}\right]\right) \\
\Phi_{i j}=k_{x} x_{i}+k_{y} y_{j}, \\
k_{x}=k \sin (\theta) \cos (\varphi), k_{y}=k \sin (\theta) \sin (\varphi),
\end{gathered}
$$

where $(R, \theta, \varphi)$ is the observation point spherical coordinates; $k$ is the free-space wavenumber; $F_{s f}$ denotes space factor [10]. Here, $F_{s f}$ includes AF, which is represented by the double exponential sum (4). Note that the aperture magnitude distribution is uniform in our case.

\section{B. Representation of Array Factor in the Presence of Phase Quantization Errors}

The beam-steering strategy is usually based on the AF optimization [6]. For the broadside plane-wave excitation, which is the case of TAs with the near-field coupling architecture, element's excitation phase can be represented in the following form:

$$
\varphi_{i j}^{\Sigma}=\varphi_{i j}^{i n}+\varphi_{i j}^{P S},
$$

where $\varphi^{i i_{i j}}$ and $\varphi^{P S} S_{i j}$ are the initial (inner) phase shift and the phase state (or the phase shifter phase) of the $(i, j)$ unit cell ( 0 or $\pi$ ), respectively. On the other hand, the value of $\varphi \Sigma_{i j}$ is determined by the desired beam-steering direction $\left(\theta_{s}, \varphi_{s}\right)$ :

$$
\begin{gathered}
\varphi_{i j}^{\Sigma}=\Phi_{i j}^{0}+\delta \varphi_{i j}, \\
\Phi_{i j}^{0}=-\left(k_{x 0} x_{i}+k_{y 0} y_{j}\right), \\
k_{x 0}=k \sin \left(\theta_{s}\right) \cos \left(\varphi_{s}\right), k_{y 0}=k \sin \left(\theta_{s}\right) \sin \left(\varphi_{s}\right) .
\end{gathered}
$$

In (8), $\delta \varphi_{i j}$ denotes the phase quantization error of $(i, j)$ unit cell due to the discrete $N$-bit phase shifter. In general, $\delta \varphi_{i j}$ can be written as

$$
\delta \varphi_{i j}=\Delta \operatorname{int}\left[\frac{\Phi_{i j}^{0}-\varphi_{i j}^{i n}}{\Delta}+0.5\right]-\left(\Phi_{i j}^{0}-\varphi_{i j}^{i n}\right)
$$

where $\Delta=2 \pi / M$ is the minimum phase increment of the TX element with $M=2^{N}$ phase states; int[-] is the integer part operator. Note that in our case of the 1-bit reconfigurable TA $N=1, M=2$.

From (4), the quantitative effect of the phase quantization on $\mathrm{AF}$ is not clear. It is convenient to represent $\mathrm{AF}$ in the form of continuous apertures superposition [9]. To implement such transformation we first use the Poisson summation formula to transfer (4) to the spectral domain. Next, we can notice that $\delta \varphi(U)$ is the periodic function of
$U=\Phi-\varphi^{i n}$. Consequently, the function $\exp (j \delta \varphi(U))$ can be represented by its Fourier series with $M=2 \pi / \Delta$ fundamental harmonic frequency. Omitting here detailed mathematical calculations, we can write $\mathrm{AF}$ in the following form:

$$
\begin{gathered}
\mathrm{AF}=\frac{1}{d_{x} d_{y}} \operatorname{sinc}(\Delta / 2) \sum_{h=-\infty}^{\infty} \frac{(-1)^{h}}{1+M h} \sum_{n=-\infty}^{\infty} \sum_{m=-\infty}^{\infty} F_{m n h}, \\
F_{m n h}=(-1)^{m\left(N_{x}-1\right)+n\left(N_{y}-1\right)} \int_{y^{(1)}}^{y^{(2)}} \int_{x^{(1)}}^{x^{(2)}} I(x, y) \mathrm{e}^{j \Phi_{m n h}} d x d y, \\
\Phi_{m n h}(x, y)=\Phi(x, y)+\Phi^{0}(x, y)+ \\
M h\left(\Phi^{0}(x, y)-\varphi^{i n}(x, y)\right)-\frac{2 \pi}{d_{x}} m x-\frac{2 \pi}{d_{y}} n y,
\end{gathered}
$$

In the given consideration, the array elements are allocated symmetrically with respect to the coordinate system origin, i.e. $x^{(2)}=-x^{(1)}=N_{x} d_{x} / 2, y^{(2)}=-y^{(1)}=N_{y} d_{y} / 2$. In (12) - (14) all continuous functions should be equal to their discrete equivalents at array grid points. The function $I(x, y)=\operatorname{rect}[x, y]$, where rect is the rectangular function over the region $x \in\left[x^{(1)}, x^{(2)}\right], y \in\left[y^{(1)}, y^{(2)}\right]$.

Despite the computational complexity of (12), this form gives us a better understanding of the phase quantization effects. Each spectral term $F_{m n h}$ represents the contribution to $\mathrm{AF}$ from the continuous aperture with $\Phi_{m n h}(x, y)$ phase distribution. The dominant term $F_{000}$ determines $\mathrm{AF}$ inside the main lobe, whereas terms with $h=0$ correspond to the conventional grating lobes. Terms with $h \neq 0$ and $m, n=0$ produce phase quantization lobes (PQLs). Finally, terms with $m, n, h \neq 0$ produce combined lobes (CLs). It is important that the magnitudes of PQLs and CLs decrease as $1 /(1+M h)$. Expressions (12) - (14) also provide an explanation on the effect of parasitic mirror lobes typical for the 1-bit phase quantization. If the 1-bit conventional TA with identical unit cells $\left(\varphi_{i j}^{i i_{i j}}=0\right)$ is excited by the plane wave, the attempt of beam steering to $\left(\theta_{s}, \varphi_{s}\right)$ direction leads to the generation of the main lobe and the aforementioned parasitic mirror lobe. The effect can be seen from (12), as in this case we have $\left|F_{00-1}\left(-\theta_{s}, \varphi_{s}\right)\right|=\left|F_{000}\left(\theta_{s}, \varphi_{s}\right)\right|$. The problem of PQL levels reduction was addressed previously in [6], [9] by using nonlinear initial phase distributions. Owing to the AF representation (12) - (14), in this contribution we can analytically study the optimum $\varphi^{i n}(x, y)$ synthesis.

\section{InITIAL PhaSe ShIFT DistribUtion SyNTHESIS}

\section{A. Virtual Focus Approach}

The conventional focal source TA architecture [6] is free from mirror lobes due to the nonlinear excitation phase distribution provided by a feeding antenna. Therefore, the first heuristic idea is to reproduce the corresponding focalsource type of phase distribution inside the TA structure. This method can be called the "virtual focus approach". In 
this case, the initial phase shift can be determined by the virtual focal length $F$ :

$$
\varphi^{i n}(x, y)=-k\left(\sqrt{x^{2}+y^{2}+F^{2}}-F\right) .
$$

For TAs of large electrical sizes, the spectral components (13) can be computed approximately using the stationary phase method [9]. For simplicity, here we will consider the 1D linear array:

$$
\begin{gathered}
F_{m h} \approx \frac{(-1)^{m\left(N_{x}-1\right)} \sqrt{2 \pi}}{\sqrt{-j d^{2} \Phi_{m h}\left(x_{0}\right) / d x^{2}}} I\left(x_{0}\right) \mathrm{e}^{j \Phi_{m h}\left(x_{0}\right)}, \\
x_{0}=\frac{F\left(k_{x}-(1+M h) k_{x 0}-2 \pi m / d x\right)}{\sqrt{M^{2} h^{2} k^{2}-\left(k_{x}-(1+M h) k_{x 0}-2 \pi m / d x\right)^{2}}} .
\end{gathered}
$$

The numerical analysis of (16) - (17) shows that the increase of $F$ leads to PQLs and CLs levels increase. At the same time, both PQLs and CLs become narrower. These considerations remain valid for $2 \mathrm{D}$ planar arrays.

Numerical simulations were elaborated to study the effect of $F$ on TA directivity degradation and SLL. Fig. 2, 3 demonstrate computed results for TAs of $8 \times 8,16 \times 16$, and $32 \times 32$ elements at different beam-steering directions in the

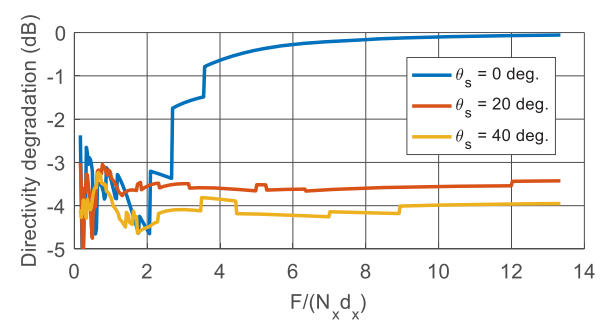

(a)

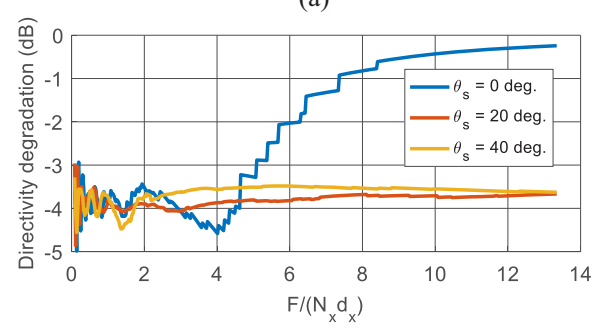

(b)

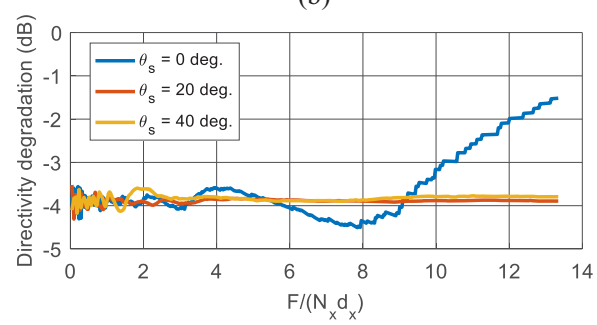

(c)

Fig. 2. Computed 1-bit tranmitarray directivity degradation as a function of the virtual focal length $F$ for different array sizes: (a) $8 \times 8$, (b) $16 \times 16$, and (c) $32 \times 32$ elements.

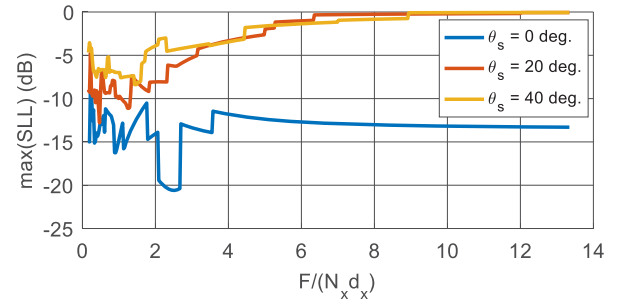

(a)

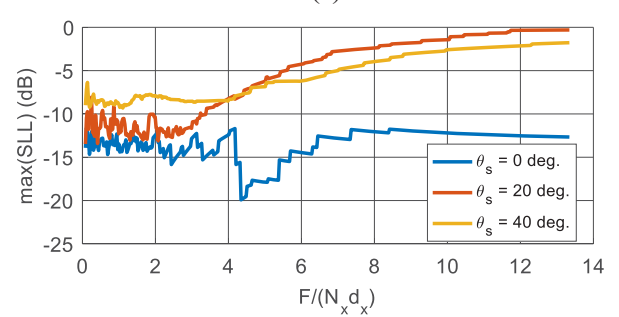

(b)

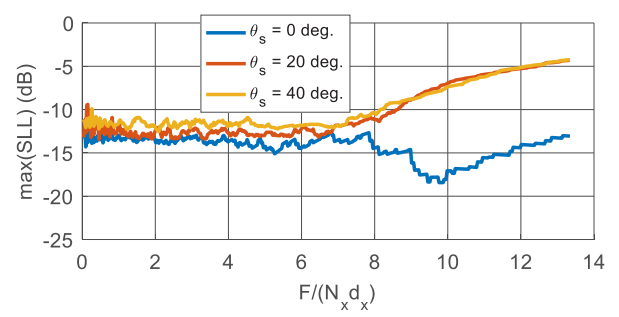

(c)

Fig. 3. Computed 1-bit tranmitarray maximum SLL in the beam steering plane as a function of the virtual focal length $F$ for dirrent array sizes: (a) $8 \times 8$, (b) $16 \times 16$, and (c) $32 \times 32$ elements.

E-plane. In all models $d_{x}=d_{y}=0.6 \lambda$, where $\lambda$ is the freespace wavelength. The directivity loss was computed by comparison with the continuous phase TA. Sidelobes performance was estimated by considering the maximum value of SLL in the beam-steering plane.

Analyzing the results, we can see that for the offbroadside beam steering, SLL goes to the $0 \mathrm{~dB}$ level as $F$ increases. This effect evidences the appearance of the parasitic mirror lobes. On the other hand, at the broadside radiation regime, the far-field pattern approaches the pattern of the uniform aperture with $-13.5 \mathrm{~dB}$ SLL. From the given data, we also can notice that the far-field performance varies rapidly for small values of $F /\left(N_{x} d_{x}\right)$, especially in the case of smaller arrays. Visual assessment of the result curves gives us the optimum (in terms of both SLL and directivity loss) value of $F /\left(N_{x} d_{x}\right)$, which is close to 1 . However, it is still hard to formulate the optimum value of $F$ analytically due to the complexity of (16) - (17).

\section{B. Optimum Initial Phase Distribution}

We look for an optimum $\varphi^{\text {in }}$ that will provide the minimum SLL. The approach is based on PQLs and CLs analysis. Let us come back to the representation of $\mathrm{AF}$ higher-order terms (16), which is valid for an arbitrary aperture field distribution. First, we require that the level of 
each lobe remain constant [9], which leads to the following equation:

$$
d^{2} \varphi^{i n}(x) / d x^{2}=-2 \pi I^{2}(x) /\left(K_{h}^{2} M|h|\right),
$$

where $\left|K_{h}\right|$ is the required constant level of the higher-order terms with index $h$. Taking into account the uniform magnitude distribution (i.e. $I(x)=\operatorname{rect}[x], x \in\left[x^{(1)}, x^{(2)}\right]$ ), and requiring the function $\varphi^{\text {in }}(x)$ to be even, the direct integration of (18) leads to

$$
\varphi^{i n}(x)=-k b_{2} x^{2},
$$

where $b_{2}=\pi /\left(k K^{2}{ }_{h} M|h|\right)$. Thus, the quadratic initial phase distribution (19) provides the same uniform level of all PQLs and CLs with index $h$. From (16) we also can find the width of each lobe $\Delta U=U_{2}-U_{1}$ :

$$
\begin{gathered}
\frac{1}{2 M|h| k b_{2}}\left(U_{1,2}-\frac{2 \pi m}{d_{x}}\right)= \pm \frac{N_{x} d_{x}}{2}, \\
\Delta U=2 d_{x} N_{x} M|h| k b_{2},
\end{gathered}
$$

where $U=\Phi_{m h}+2 \pi m / d_{x}$. At the same, the CLs with index $h$ are repeated along $U$-axis with the period $2 \pi m / d_{x}$. If the value of $b_{2}$ increases then PQLs and CL levels decrease, while the lobes become wider. In [9], authors supposed that the optimum value of $b_{2}$ corresponds to the situation when for some particular index $h$ the lobes are as small as possible but still do not overlap. As the lobes with $h= \pm 1$ have the strongest level, the optimum value $b_{2 o p t}$ can be found as

$$
b_{2 o p t}=\lambda /\left(2 N_{x} d_{x}^{2} M\right) \text {. }
$$

It is worth mentioning that the presented synthesis procedure can be extended directly to the $2 \mathrm{D}$ case:

$$
\varphi^{i n}(x, y)=-k\left(b_{2 x} x^{2}+b_{2 y} y^{2}\right),
$$

where the "optimum" values of $b_{2 x / y}$ are given by (22) with the appropriate parameters substitution. The aforementioned optimum criterion of nonoverlapping lobes should be carefully verified during the numerical experiments. For this reason, the same far-field performance metrics as in Section II.A was computed for different values of $b_{2 x}=b_{2 y}$. The results are presented in Fig. 4, 5. Note that the virtual focus phase distribution (15) is very close to the quadratic distribution (23) for $F=1 /\left(2 b_{2 x}\right)$ if $2 F /\left(N_{x} d_{\mathrm{x}}\right)>1$. Due to this reason, in Fig. 4, 5 the far-field performance is plotted versus $1 /\left(2 b_{2 x} N_{x} d_{x}\right)$. In these plots, the vertical dashed line denotes the optimum value of $b_{2 x / y}$ according to (22). From Fig. 5 , we can see that $b_{2 o p t}$ indeed corresponds to some local minima of the $\max (\mathrm{SLL})$ function for all beamsteering directions. For high values of the argument, the virtual focus and the quadratic phase distribution curves become similar. The substantial discrepancy is observed for small values of $F$, when $2 F /\left(N_{x} d_{\mathrm{x}}\right)<1$. At the same time, $b_{2 o p t}$ corresponds to $F_{\text {opt }}=1 /\left(2 b_{2 o p t}\right)$. The ratio $2 F_{\text {opt }} /\left(N_{x} d_{\mathrm{x}}\right)=2 M d_{x} / \lambda>1$, which means that the far-field performance of two initial phase distributions should be close for these parameters values. We can actually see it comparing the result. Thus, a more precise comparison is required to clarify the available far-field performance improvement.

\section{Far-Field Patterns Comparison}

Far-field patterns of various TA configurations were studied numerically for both initial phase distributions. Some results are presented in Fig. 6, where patterns are normalized to the absolute maximum of all curves. Application of the virtual focus and the quadratic initial phase distributions with optimum parameters provide similar SLL performance. Moreover, simulations predict that for relatively small TAs, the virtual focus approach can result in even lower level of the first sidelobes. We extended the analysis considering different values of $b_{2 x / y}$ and found that for $N_{x}, N_{y}=2^{\mathrm{Q}}$, where $\mathrm{Q}$ is a natural number, a substantial improvement in the average SLL can be achieved if $b_{2 x / y}=2 b_{2 o p t}$. The corresponding patterns are shown in Fig. 6. The SLL improvement was observed for various array sizes, as well as for different $\theta_{s}$. The reported effect most likely comes from the destructive interference

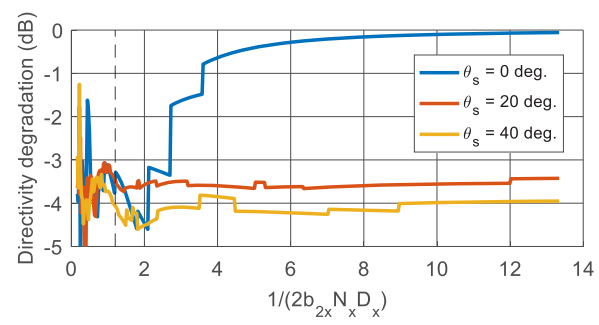

(a)

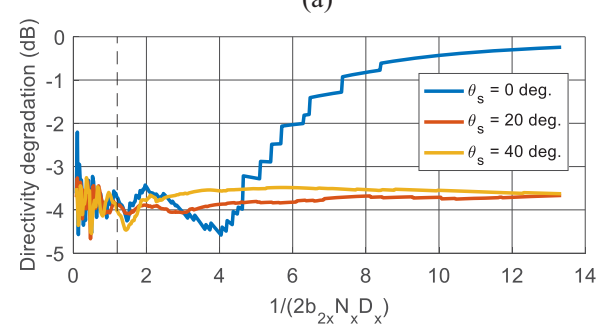

(b)

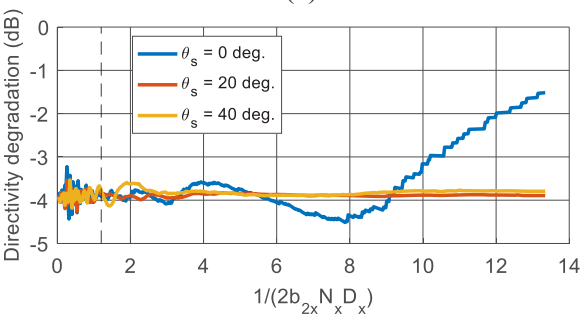

(c)

Fig. 4. Computed 1-bit tranmitarray directivity degradation as a function of $b_{2 x}$ parameter value for different array sizes: (a) $8 \times 8$, (b) $16 \times 16$, and (c) $32 \times 32$ elements. 


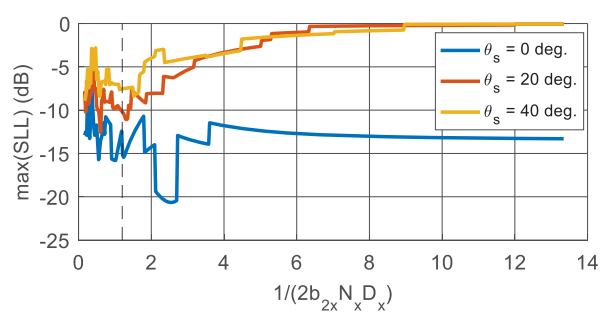

(a)

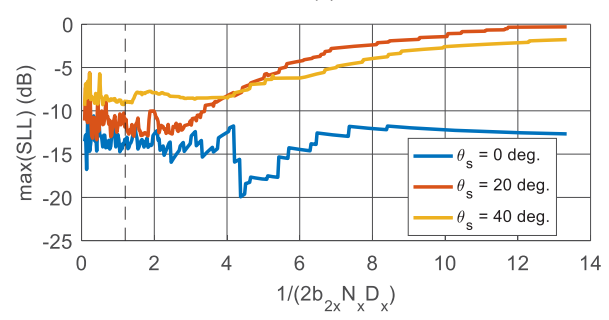

(b)

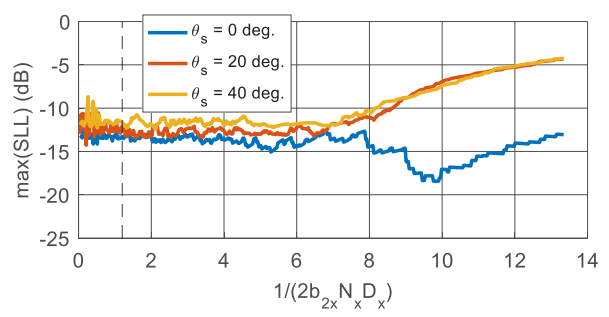

(c)

Fig. 5. Computed 1-bit tranmitarray maximum SLL in the beam steering plane as a function of $b_{2 x}$ parameter value for different array sizes: (a) $8 \times 8$, (b) $16 \times 16$, and (c) $32 \times 32$ elements.

between overlapping higher-order PQLs and CLs determined by the phase $\Phi_{m h}\left(x_{0}\right)$ in (16). However, it should be noted that the SLL improvement is more narrowband compared to the optimum parameter value. This interference requires some further mathematical investigation and is out of the scope of the present work.

\section{CONCLUSION}

In this contribution, we provided the detailed analysis of the effects arising in 1-bit TAs with the near-field coupling feeding technique. The employed AF representation allows the analytical formulation of the optimum initial phase distribution. We demonstrated that the virtual focus and the optimum quadratic initial phase distributions are very close in terms of maximum SLL performance. The results of this work can be useful for the initial stage of 1-bit phased antenna arrays development. Further research efforts are believed may lead to an additional improvement of SLL performance due to the effect of PQLs and CLs destructive interference.

\section{REFERENCES}

[1] S. V. Hum and J. Perruisseau-Carrier, "Reconfigurable reflectarrays and array lenses for dynamic antenna beam control: a review," IEEE Trans. Antennas Propag., vol. 62, no. 1, pp. 183-198, Jan. 2014.

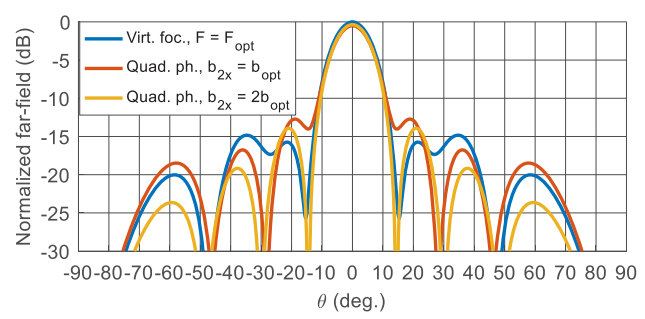

(a)

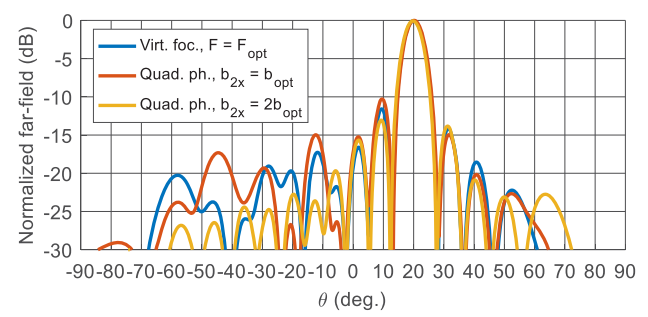

(b)

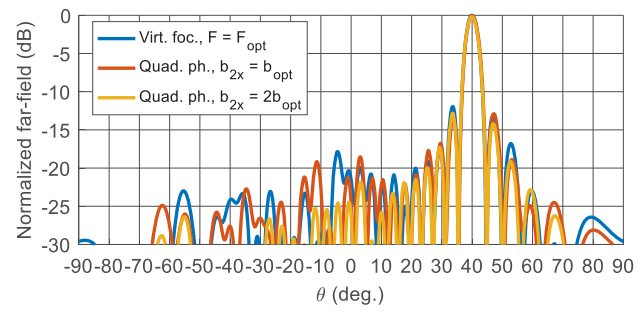

(c)

Fig. 6. Computed normalized far-field patterns of 1-bit tranmitarray for different array configurations: (a) $8 \times 8, \theta_{s}=0^{\circ}$; (b) $16 \times 16, \theta_{s}=20^{\circ}$; (c) $32 \times 32, \theta_{s}=40^{\circ}$

[2] A. Clemente, L. Dussopt, R. Sauleau, P. Potier, and P. Pouliguen, "Wideband 400-Element electronically reconfigurable transmitarray in X band," IEEE Trans. Antennas Propag., vol. 61, no. 10, pp. 50175027, Oct. 2014.

[3] J. G. Nicholls and S. V. Hum, "Full-space electronic beam-steering transmitarray with integrated leaky-wave feed," IEEE Trans. Antennas Propag., vol. 64, no. 8, pp. 3410-3422, Aug. 2016.

[4] B. D. Nguyen and C. Pichot, "Unit-cell loaded with PIN diodes for 1bit linearly polarized reconfigurable transmitarrays," IEEE Antennas Wireless Propag. Lett, vol. 18, no. 1, pp. 98-102, Jan. 2019.

[5] L. Di Palma, A. Clemente, L. Dussopt, R. Sauleau, P. Potier, and P. Pouliguen, "Circularly-polarized reconfigurable transmitarray in Ka-band with beam scanning and polarization switching capabilities," IEEE Trans. Antennas Propag., vol. 65, no. 2, pp. 529-540, Feb. 2017.

[6] R. J. Mailloux, Phased array antenna handbook, 3rd ed., Norwood, MA: Artech House, 2018.

[7] M. S. Smith and Y. C. Guo, "A comparison of methods for randomizing phase quantization errors in phased arrays," IEEE Trans. Antennas and Propag., vol. AP-31, no. 6, pp. 821-828, Nov. 1983.

[8] H. Kamoda, J. Tsumochi, T. Kuki, and F. Suginoshita, "A study on antenna gain degradation due to digital phase shifter in phased array antennas," Microwave and Optical Technology Letters, vol. 53, no. 8, pp. 1743-1746, Aug. 2011.

[9] D. I. Voskresenskiy, L. I. Ponomarev, and V. S. Filippov, Convex scanning antennas: basic theory and analysis methods, Moscow: Soviet Radio, 1978. (In Russian)

[10] C. K. Balanis, Antenna Theory: Analysis and Design. 3rd ed., Hoboken, NJ: John Wiley \& Sons, Inc., 2005. 